Support information

\title{
Acoustic bubble suppression by constructing a hydrophilic coating on HDPE surface
}

Yuemei Ye ${ }^{1}$, Stanislav Klimchuk ${ }^{1}$, Mingwei Shang ${ }^{1}$, Kenneth McDonald ${ }^{2}$, Junjie Niu ${ }^{1 *}$

${ }^{1}$ Department of Materials Science and Engineering, CEAS, University of Wisconsin-Milwaukee Milwaukee, WI 53211

${ }^{2}$ Controls Technologies Division, SSI Technologies, Inc.

Plymouth, MI 48170

*Corresponding author e-mail: niu@uwm.edu (J Niu)

Figure S-1

Figure S-2

Figure S-3

Figure S-4 

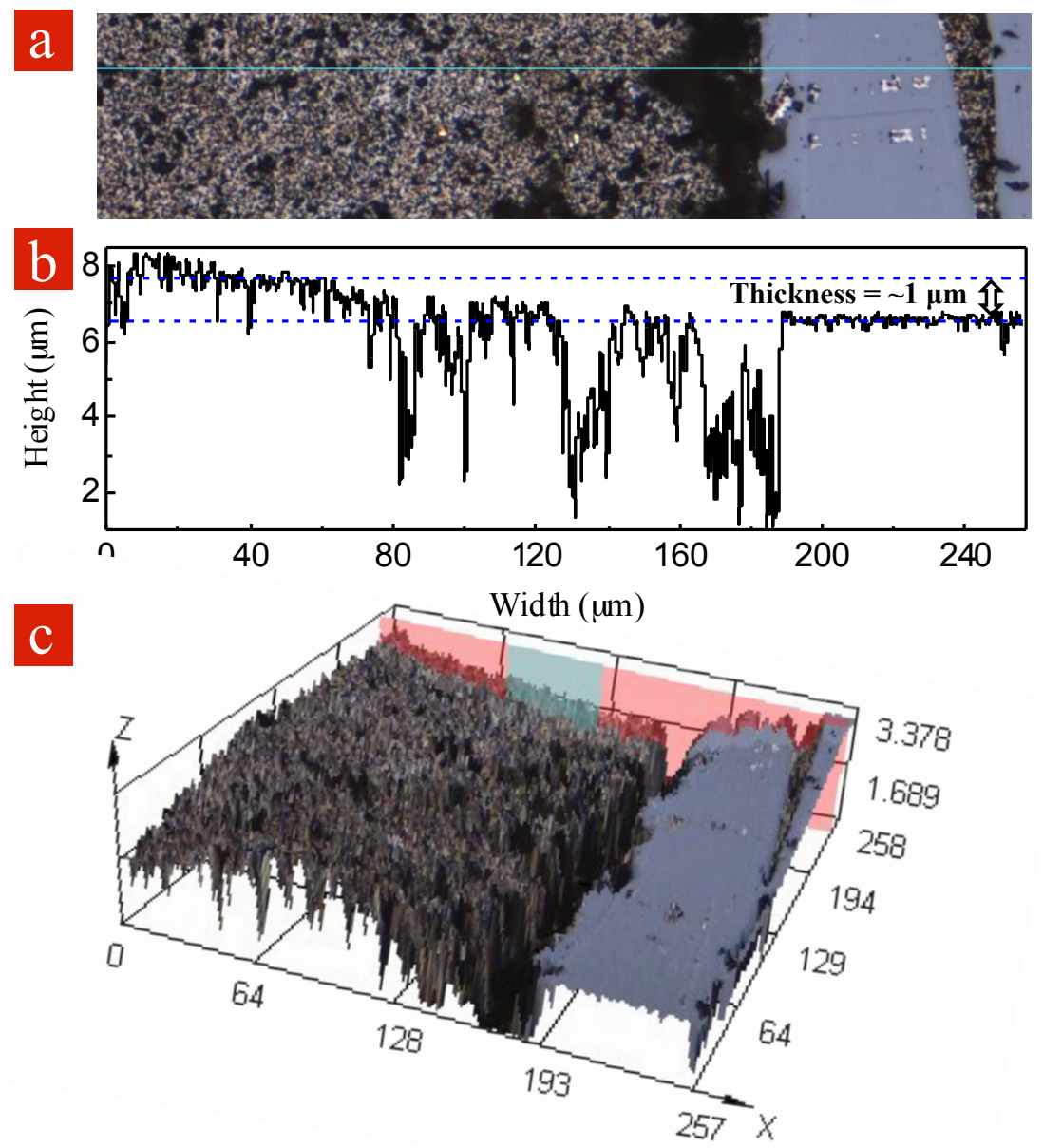

Figure S-1. The surface (a) and 3D roughness (c) morphologies of the polydopamine coated glass by confocal optical microscopy. (b) Thickness measurement of the coating in (a). 


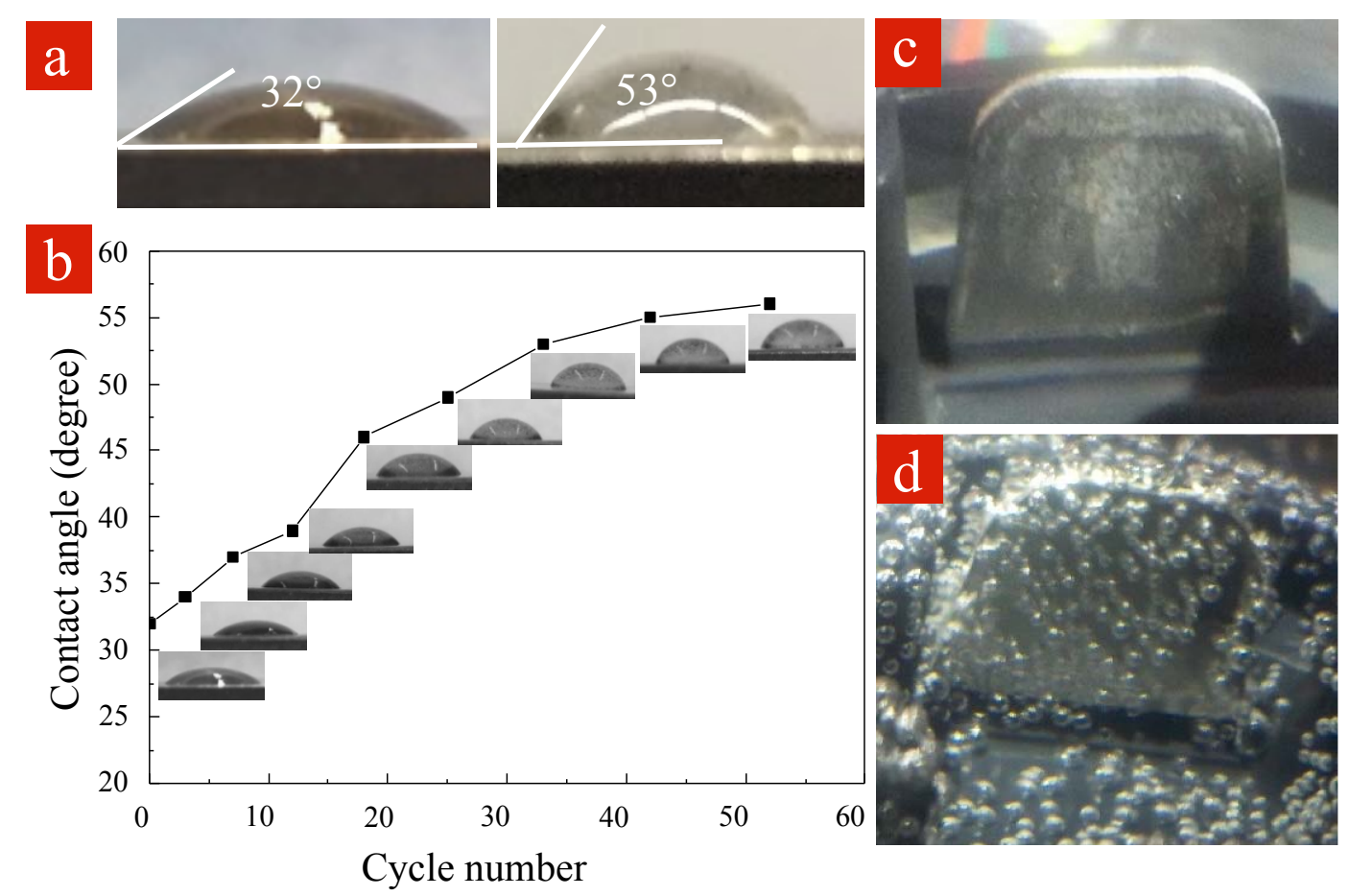

Figure S-2. (a) Water contact angle of the polydopamine coated (left) and uncoated (right) stainless steel reflectors dissembled from UTC sensors. (b) Contact angle evolution of the polydopamine coated reflector as a function of abrasion cycle under a pressure of $9.8 \mathrm{kPa}$. Bubble removal performance of the polydopamine coated (c) and uncoated (d) reflectors from UTC sensors after ultrasonic agitation in DI water at $60{ }^{\circ} \mathrm{C}$ for $4 \mathrm{~h}$. 
Coated

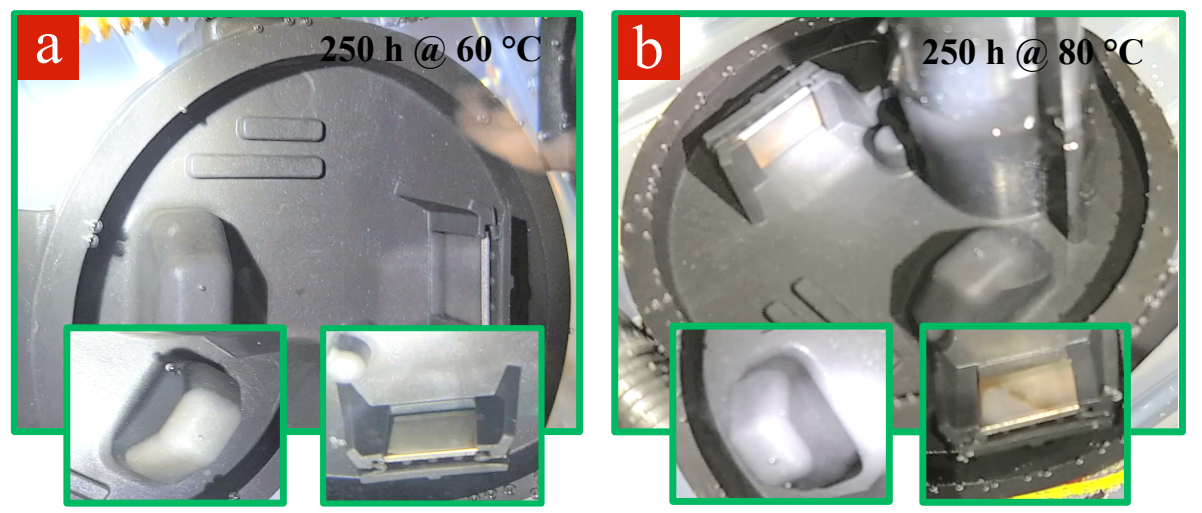

Tombstone

Reflector

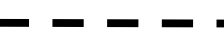

Uncoated
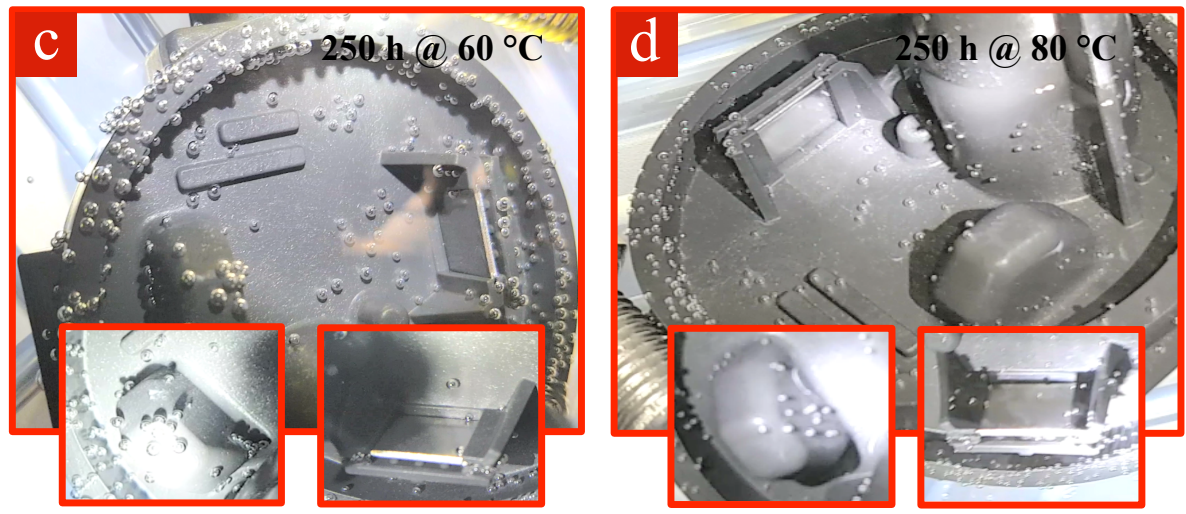

Figure S-3. Anti-aeration nucleation tests of the polydopamine coated (a) and uncoated (c) primer pretreated UTLC sensors after ultrasonic agitation in DEF solution at $60^{\circ} \mathrm{C}$ for 250 hours. Anti-aeration nucleation tests of the polydopamine coated (b) and uncoated (d) primer pre-treated UTLC sensors after ultrasonic agitation in DEF at $80^{\circ} \mathrm{C}$ for 250 hours. 

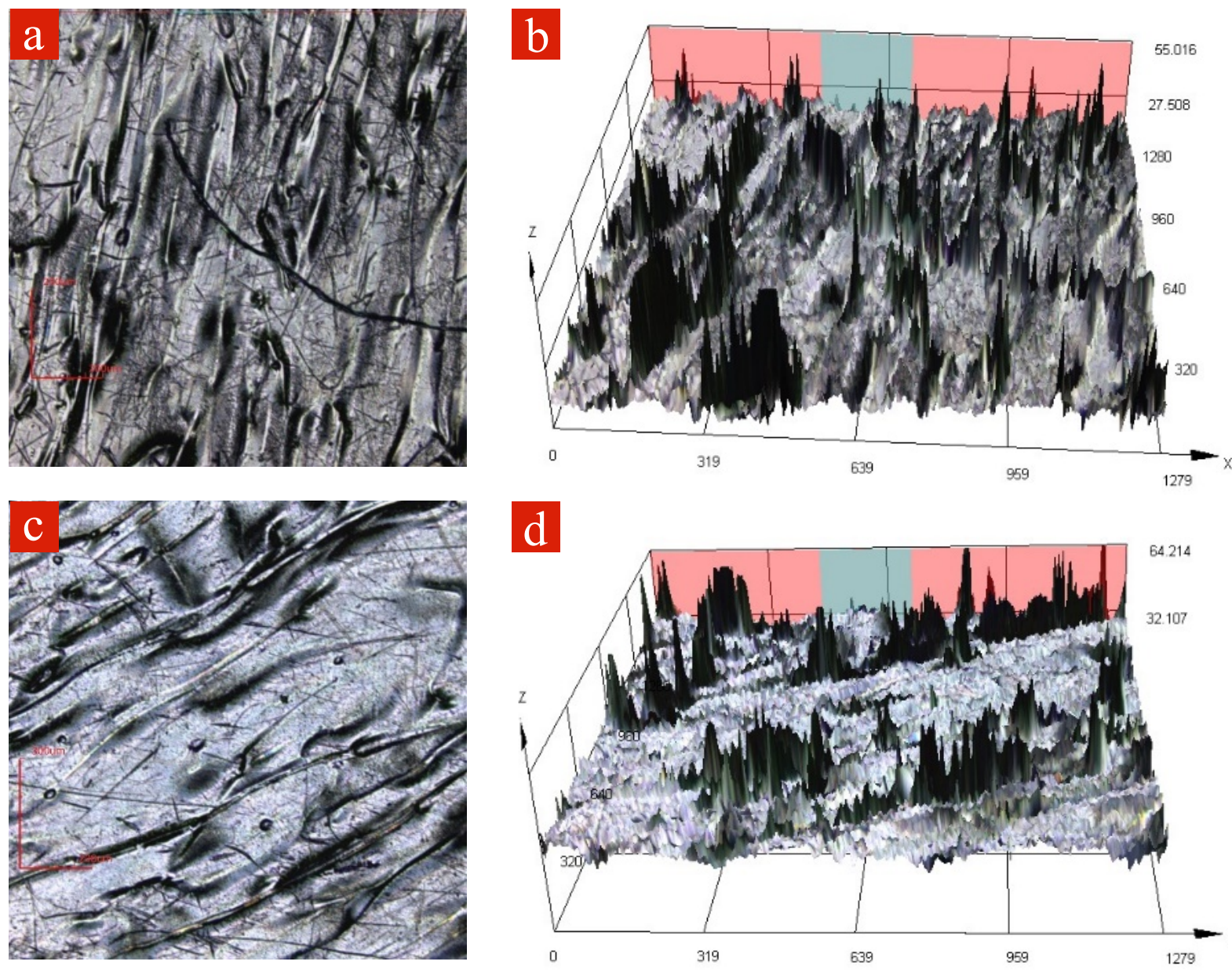

Figure S-4. The surface and 3D roughness morphologies of the HDPE (a, b) and polydopamine coated HDPE (c, d) by confocal optical microscopy. 